\title{
Fuzzy Reasoning Model To Improve Face Illumination Invariance
}

\author{
Adel Oulefki $^{1}$. Mustapha Aouache ${ }^{1}$. Elhocine \\ Boutellaa $^{2}$. Messaoud Bengherabi ${ }^{1}$. Ahmed \\ Amine Tifarine ${ }^{1}$
}

the date of receipt and acceptance should be inserted later

\begin{abstract}
Enhancing facial images captured under different lighting conditions is an important challenge and a crucial component in the Automatic Face Recognition Systems (AFRS). We tackle this problem by proposing a new face image enhancement approach based on Fuzzy theory. Depending on the illumination of a given image, the Fuzzy-logic generates an adaptive factor which is used for correcting the illumination. The proposed approach improves non-uniform illumination and low contrasts, often encountered during capturing process in severe environmental conditions. Our approach is assessed using four blind-reference image quality metrics as well as visual assessment. A comparison to six state-of-the-art methods is provided. Experiments are performed on four public data sets, namely EYale-B, Mobio, FERET and CMU-PIE, showing very interesting results achieved by our approach.
\end{abstract}

Keywords Fuzzy System · Fuzzy Filter · Image Enhancement · Image quality measurement $(\mathrm{IQM}) \cdot$ Face analysis

\section{Introduction}

Automatic Face Recognition Systems (AFRS) have received a significant attention from the biometric community over the past several decades owing to their wide range of applications in information security and access control, law enforcement, surveillance, etc. [21]. The AFRS attain convincing performances when dealing with face images captured under controlled conditions. However, the recognition performances degrade dramatically in uncontrolled environments.

One of the most challenging problems to AFRS is illumination variations. To deal with illumination change, image enhancement methods are applied to face images aiming to improve the face clarity and to bring out the hidden features. A wide

\footnotetext{
${ }^{1}$ Centre de Dévelopment des Technologies Avancées, Baba Hassen, Algeria

${ }^{2}$ Center for Machine Vision and Signal Analysis, University of Oulu, Oulu, Finland
} 
spectrum of image enhancement approaches has been proposed in the literature. Histogram Equalization (HE) is a widely used image enhancement technique as it is efficient, simple to implement and fast [15]. HE preserves the mean brightness of the resulting image. However, saturation effects may appear in both bright and dark intensity values, giving the impression unnatural look to the enhancement [1]. Inspired by HE, Many enhancement approaches have been proposed.

One improved version of the HE method is Contrast Limited Adaptive Histogram Equalization (CLAHE). Compared to HE, CLAHE decomposes an input image into blocks, rather than using the entire image. Contrast enhancement is then applied to each block independently. The resulting enhanced blocks are finally recombined and bi-linear interpolation is employed in order to eliminate artificially induced boundaries [13]. The drawback of the CLAHE algorithm is that the noise in the homogeneous blocks is increased as the contrast is increased.

In another work, Poddar et al. [14] suggested a Non-parametric Modified Histogram Equalization for Contrast Enhancement (NMHE). This approach removes spikes from the histogram of the input image. Then the histogram is clipped and normalized. A new histogram is computed as a weighted mean of the modified histogram and the uniform histogram. The weight is the summed deviation of the intermediate modified histogram from the uniform histogram. Contrast enhancement is then achieved by using the distribution function of the new obtained histogram as the transformation function.

Similar to the previous techniques, Arriaga-Garcia et al. in [4] introduced Bihistogram Equalization based on Adaptive Sigmoid Function (BEASF) for image contrast enhancement. The image enhancement is achieved via brightness preservation and noise tolerance. First the image histogram is split into two sub-histograms applying the mean as a threshold and their cumulative distribution functions are replaced by two smooth Sigmoids (with their origins placed on the median of each sub-histogram). Afterward, a HE is performed and followed by histogram stretching within their own limits resulting in a continuous and smooth mapping curve.

Image enhancement has also been explored in the frequency domain using the wavelet transform (WA) [6]. WA is applied to the original image to generate its low frequency and high frequency components. This aims to manipulate different components separately. Histogram equalization is applied to the low frequency while high frequency is multiplied by a scalar. The former operation refines the approximation whereas the latter one accentuates the detail. Finally, the inverse wavelet transform is applied to the modified coefficients, resulting into the normalized image. WA simultaneously enhances the contrast and edges of face images in the frequency domain.

Fuzzy logic based image enhancement has been proposed by Sheet et al. [16], who proposed Brightness Preserving Dynamic Fuzzy Histogram Equalization (BPDFHE). The BPDFHE uses a Fuzzy histogram of the image to handle the inexactness of gray level values. operates the image histogram in order to prevent remapping the histogram peaks. Therefore, a redistribution of the gray-level values in the valley portions between two consecutive peaks of the histogram is performed. Under different illuminations, BPDFHE produce well-enhanced contrast with a few artifacts. This method has the drawbacks of requiring the algorithm complexity and high computation time. 
All the aforementioned image enhancement techniques suffer from enhancement imperfection. In order to tackle these issues, this paper proposes a new enhancement approach based on Fuzzy-logic for face images with the non-uniform illumination and low contrast. Depending on the illumination of a given image, the Fazzy-logic generates an adaptive factor which is used for correcting the illumination. While the conventional approaches fail, the proposed approach preserves the brightness and increases the clarity of the face. Thus, more face features can be easily appear. The simulation results will show that the proposed fuzzy image enhancement approach has good results comparing to the state-of-the-art.

This paper is organized as follows. Section 2 presents the proposed fuzzy image enhancement technique. Section 3 explains the employed evaluation procedure in terms of visual representation and quantitative measurement; provides the experimental results; and shows the effectiveness of the proposed fuzzy image enhancement technique compared to other alternative schemes, such as histogram equalization and contrast limited adaptive histogram equalization. Section 4 provides the conclusions of this work based on the conducted analysis.

\section{Proposed approach}

The conventional image enhancement methods suffer from some imperfections such us over/less enhancement, noise amplification and also some artifacts effect take place. When applied to face images, the enhancement often generates unnatural appearance and unreliable data for AFRS. Therefore, we propose a new approach to tackle the previous issues. On one hand, the proposed approach aims to improve both the contrast and the brightness of the original image. On the other hand, it brings out the hidden details of the face images. The general framework of the proposed method based face details and discusses given as in Figure 1

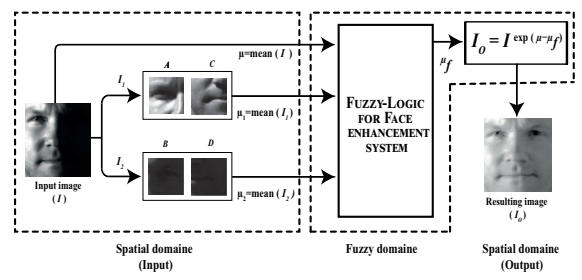

Fig. 1: Overview of the proposed face image illumination enhancement technique based on the Fuzzy-logic system.

Fuzzy-logic is a computational paradigm which provides a mathematical solution and a representation, along with manipulating information in such a way to resemble to human reasoning process [19]. In our work, we employ a Fuzzy-logic system for solving the problem of face images illumination variation. Mainly, as any other Fuzzy approach, the Fuzzy image enhancement consists of three fundamental stages [20], namely (i) image fuzzification, (ii) extract membership value for the enhancement 
process, (iii) ending with the process of image defuzzification [18]. The proposed method is a rule-base for face enhancement technique which has three inputs and one output as shown in in Figure 1. The original input image $I$ is divided into four equal parts, each part labeled as: A, B, C and D. The four face parts represent the upper left part, the upper right part, the lower left part and the lower right part, respectively. The inputs of the Fuzzy-logic system are $\mu, \mu_{1}$ and $\mu_{2}$ standing for the mean images intensity computed from the original images, parts (A, C) and parts (B, D). These inputs symbolize the illumination of a given image based on its intensity values. The Fuzzy-logic is employed to derive an adaptive factor $\mu_{f}$. This factor is then used for a nonlinear transformation of the image, aiming to correct its illumination. The proposed Fuzzy system is defined by seven Fuzzy sets based on the mean intensity of the input images. The Fuzzy sets and their nomination used in this study are defined in Table ??. Shown in Figure ??, the Gaussian membership functions of the seven

Table 1: Designation of the Fuzzy sets used in our approach.

\begin{tabular}{c||c}
\hline The Fuzzy sets base & Abbreviation \\
\hline High Dark & $\mathrm{HD}$ \\
Moderate Dark & $\mathrm{MD}$ \\
Slight Dark & $\mathrm{SD}$ \\
Uniform & $\mathrm{U}$ \\
Slight Bright & $\mathrm{SB}$ \\
Moderate Bright & $\mathrm{MB}$ \\
High Bright & $\mathrm{HB}$ \\
\hline
\end{tabular}

Fuzzy sets representing the inputs and the resulting Fuzzy output $\left(\mu_{f}\right)$, respectively. The inference engine used for the proposed Fuzzy face illumination enhancement

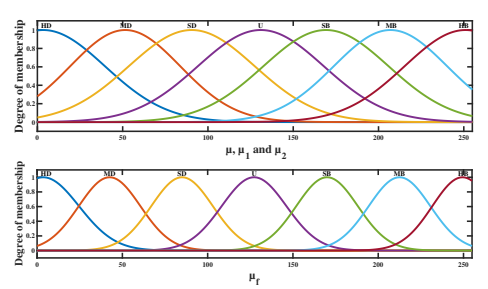

Fig. 2: Top: Gaussian membership functions of the proposed Fuzzy face enhancement. The same membership functions are used for the three inputs $\mu, \mu_{1}$ and $\mu_{2}$. Bottom: the Gaussian membership functions of the Fuzzy output $\left(\mu_{f}\right)$.

system is a 'mamdani', max-min method and centroid as defuzzification. The Fuzzy rule-base of the proposed system is borrowed from a tracking problem related to control theory. Figure ?? presents the Fuzzy rule-base surface viewer of the inputs $(\mu),\left(\mu_{1}\right)$ and $\left(\mu_{2}\right)$ versus output $\left(I_{O}\right)$. The rules are generated to preserve the quality 
of the input face images in both bright and dark regions. In the proposed method, we applied Fuzzy contrast manipulation technique by using exponential function. The pixels intensity are changed adaptively using an input-output transformation that varies based on local image characteristics. By the specified definition, the Fuzzy rule-base could be presented as follows:

Fuzzy rules:

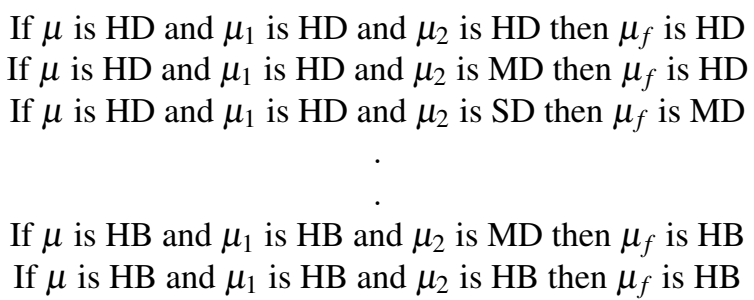

Using $\mu$ as input to the Fuzzy system, $\mu_{f}$ is the gain factor generated from the Fuzzy output. Then the resulting face image given as Equation in ?? :

$$
I_{O}=I^{\exp \left(\mu-\mu_{f}\right)}
$$

where $I$ is the input image, and $\mu$ represent the mean intensity of $I . \mu_{f}$ is the resulting value from the Fuzzy system.

Depending on the illumination of the input image regions, the particular rules are eliminated. As the input is distributed with overlap Fuzzy sets, the proposed enhancement technique significantly reduces artifact and preserves the face shape (e.g: eyebrows, nose, nostril and mouth). It also produces a uniform texture for each $I_{O}$ output image.

\section{Results and discussion}

In this section we evaluate the proposed approach using several statistical measurements as well as via visual assessment of the enhanced face images. To this end, four public databases containing face images with non-uniform illumination, namely EYale-B, Mobio, FERET and CMU-PIE are used. Additionally, for the sake of comparison, all the experiments are also performed on six state-of-the-art enhancement approaches, specifically, Histogram Equalization (HE), Contrast Limited Adaptive Histogram Equalization (CLAHE), Brightness Preserving Dynamic Fuzzy HE (BPDFHE), Wavelet based enhancement (WA), Non-parametric Modified HE (NMHE) and Bihistogram Equalization based on Adaptive Sigmoid Function (BEASF).

\subsection{Face databases}

The four face databases used for the evaluation of the proposed method are presented in the following.

Extended Yale B Face Database (EYale-B) [10] contains 16,128 images of 38 human subjects captured under 9 poses and 64 illumination conditions. The database 
extends the original one [8] by involving 28 new subjects. Five different light sources positioned at 12, 25, 50, 77and 90 degrees of the camera axis are employed for capturing the faces under illumination variation. For each subject in every particular pose, an image with ambient (background) illumination was also captured.

FERET database [12] was collected through 15 different sessions between August 1993 and July 1996, each session lasted one to two days. The database involved 1,199 individuals. For each individual 5 to 11 images were captured. In total, the FERET database contains 14,126 face images. The images present different challenges including person aging, facial expressions and illumination change.

Mobio database [11] contains talking face videos of 152 people. The gender statistics of the database participants are 100 males and 52 females. The database recording was performed using two types of mobile devices: mobile phones (NOKIA N93i) and laptop computers (standard 2008 MacBook). The database was collected on a period of about 2 years from August 2008 until July 2010 in six different universities sites. The recording environment was uncontrolled involving variations in illumination, facial expression, and face pose. The data of each individual was captured in 12 sessions to maximize the intra-person variability. In our work, we use the facial still images of the Mobio database. The images are extracted from each video by the database collectors.

CMU Pose, Illumination, and Expression database (CMU-PIE) [17] contains over 40,000 facial images of 68 people. For each person, many images are captured under 13 different poses, 43 different illumination conditions, with 4 different expressions. The database was collected over a period of three months. The recording was performed in a dedicated room using 13 high quality Sony DXC 9000 cameras. The images are color ones with a resolution of $640 \times 486$. The illumination has been generated using a flash system of 21 different flashes.

\subsection{Image quality metrics (IQM)}

IQM aim to statistically model and measure the image quality by providing quantitative values. To evaluate the proposed approach and compare it with other contemporary enhancement methods. Four IQM measures are selected as: i) EME (enhancement by entropy), ii) SDME (second-derivative-like measure of enhancement) iii) Sharpness (SHRP), and iv) CPP (contrast per pixel). These four measures are briefly described below.

i. EME has been proposed by Agaian et al. [2] [3]. Basically, it provides an absolute score to each image by measuring its contrast using Webers law. Then the contrast is related to the perceived brightness according to Fishers law. Formally, the EME is defined by:

$$
E M E=\chi\left(\frac{1}{k_{1} k_{2}} \sum_{i=1}^{k_{1}} \sum_{j=1}^{k_{2}} 20 \ln \left[\frac{I_{\text {max } ; k, l}^{w}}{I_{m i n ; k, l}^{w}+c}\right]\right)
$$

where, the evaluated image $I$ is separated into $k_{1} k_{2}$ blocks $w_{k, l}(i, j)$ of size $l_{1} \times$ $l_{2}$, with $\mathrm{c}$ is a constant which is generally set to $0.0001, I_{\min }$ and $I_{\max }$ are the 
minimum and maximum values of the pixels in each block. Function $\chi$ is sign function, $\chi(x)=x$ if $x \geq 0$, else $\chi(x)=-x$.

ii. SDME use the second derivative [22] for measuring the image quality. While EME only takes into account minimum and maximum pixel values, in addition to that, SDME consider also the center pixel value. This is less sensitive to noise and steep edges compared to the EME measure [22]. The SDME is computed as follows:

$$
S D M E=-\frac{1}{k_{1} k_{2}} \sum_{i=1}^{k_{1}} \sum_{j=1}^{k_{2}} 20 \ln \left[\frac{I_{\text {max } ; k, l}-2 I_{\text {center } ; k, l}+I_{\text {min } ; k, l}}{I_{\max ; k, l}+2 I_{\text {center } ; k, l}+I_{\text {min } ; k, l}}\right]
$$

where $I_{\text {center }}$ present the center pixel value of each block.

iii. SHRP is a measure that shows the capacity of a filter to steadily reduce the noise [9] and computed as the mean of intensity differences between adjacent pixels in both vertical and horizontal directions:

$$
\begin{aligned}
S H R P & =\frac{1}{2}\left[\frac{1}{(N-1) M} \sum_{i=1}^{M} \sum_{j=1}^{N-1}\left|I_{i, j}-I_{i, j+1}\right|\right. \\
& \left.+\frac{1}{(M-1) N} \sum_{i=1}^{M-1} \sum_{j=1}^{N}\left|I_{i, j}-I_{i+1, j}\right|\right]
\end{aligned}
$$

where, $I$ denotes an image of size $M \times N$.

iv. CPP as in [7] [5] is an estimation of the average intensity difference between a pixel and its neighboring pixel. The CPP of an image is defined as:

$$
C P P=\frac{\sum_{i=0}^{M} \sum_{j=0}^{N}\left(\sum_{(m, n) \in R_{3}^{(i, j)}}|I(i, j)-I(m, n)|\right)}{M N}
$$

where, $I(i, j)$ is the gray value of pixel $(i, j), R_{3}^{(i, j)}$ is a window of $3 \times 3$ centered at pixel $(i, j)$.

\subsection{Blind-reference assessment}

To demonstrate the robustness of our proposed enhancement method (FEA), quantitative assessments are computed and compared with six literature enhancement methods (i.e., HE, CLAHE, BPDFHE, NMHE, WA and BEASF). Therefore, for each enhancement approach, we compute the IQM using the four face databases (EYale-B, Mobio, FERET, and CMU-PIE). Following, we report the results with details.

Plots in Figures 2, 3, 4 and 5 are the error bars generated from the average and standard deviation of the EME, SDME, SHRP, and CPP metrics, respectively; of different enhancement techniques using EYale-B, Mobio, FERET and CMU-PIE data sets. The error bars provide a better and simpler representation of the quantitative metric by depicting the average and standard deviation of each metric on a given database. 

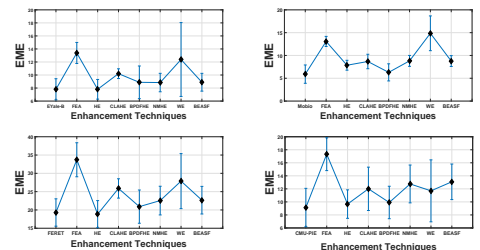

Fig. 3: Error bars (mean and standard deviation) computing from the EME resulting metric of different enhancement techniques using EYale-B, Mobio, FERET and CMU-PIE data sets.
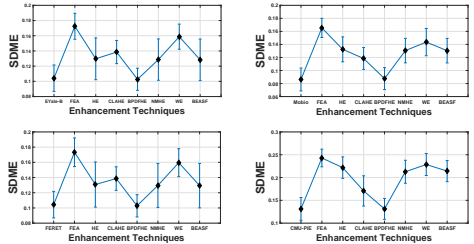

Fig. 4: Error bars (mean and standard deviation) computing from the SDME resulting metric of different enhancement techniques using EYale-B, Mobio, FERET and CMU-PIE data sets.
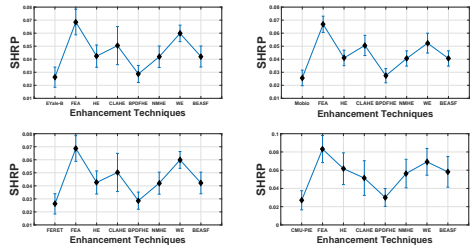

Fig. 5: Error bars (mean and standard deviation) computing from the SHRP resulting metric of different enhancement techniques using EYale-B, Mobio, FERET and CMU-PIE data sets. 


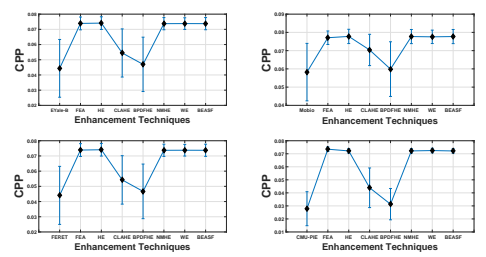

Fig. 6: Error bars (mean and standard deviation) computing from the CPP resulting metric of different enhancement techniques using EYale-B, Mobio, FERET and CMU-PIE data sets.

The provided results in the table and figures clearly show that our proposed approach outperforms the state of the art in most cases. In terms of EME, the only case where our approach has been beaten is on the Mobio data set by the WA approach. However, we note that our approach is more stable since the standard deviation of WE tends to be much larger than it is for our approach.

In terms of SDME and sharpness our approach gives the best results on the four data sets. These results indicate that FEA significantly sharpen and enhance the original image. Therefore, it provides faces with more details. Finally, the CPP metric demonstrate that our approach achieves competitive results compared to HE, NMHE, BEASF and WA while it is significantly better than CLAHE and BPDFHE. This last result proves the ability of the proposed method to improve the input image contrast.

The above observations reveal that our approach achieves better image enhancement (EME \& SDME) and sharpness (SHRP) while it competes the other approaches in contrast enhancement (CPP). The results of our approach have highest average and lowest standard deviation showing its effectiveness and stability.

\subsection{Visual assessment}

Robust image enhancement techniques are expected to significantly improve the quality of the face images, meanwhile, preserving the face details. Furthermore, both bright and dark regions should be enhanced, when artifacts ought not to be amplified. In this subsection, a qualitative comparison between our enhancement technique and the six state-of-the-art ones is conducted. Over and less enhancement are the most common drawbacks of the existing image enhancement techniques. Enhancement methods often tend either to over or less enhance the foreground and/or the background of the input images when unnatural effects appear in different areas. The qualitative assessment aims demonstrate the potential of the proposed Fuzzy-based image enhancement technique to overcome the mentioned issues.

In Figures 6, 7, 8 and 9, we depict the results of applying the enhancement methods on face samples taken from EYale-B, Mobio, FERET and CMU-PIE data sets, respectively. The face samples are chosen so that the lightning varies from the darkest to the brightest. 
Firstly, considering the darkest illumination case shown in Figure 6, it is obvious that BEASF and WA over enhance the image presenting more undesirable effects, meanwhile NMHE and HE yield over-brightness, particularly in the bright regions. Regarding CLAHE and BPDFHE approaches, one can notice that these methods less enhance the too dark input images.

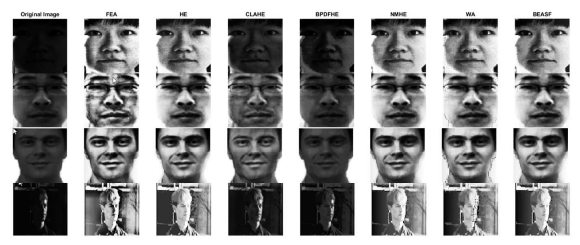

Fig. 7: Example of darkest face image enhancement using our approach (FEA), HE, CLAHE, BPDFHE, NMHE, WA and BEASF. From the top row to the bottom row, face samples belong to EYale-B, Mobio, FERET and CMU-PIE, respectively.

In the case of face image with a slight darkness, Figure 7, we can see that HE, BEASF and NMHE are steadily competitive, but a non-uniform lighting appear. CLAHE and BPDFHE still suffer from poor visibility.

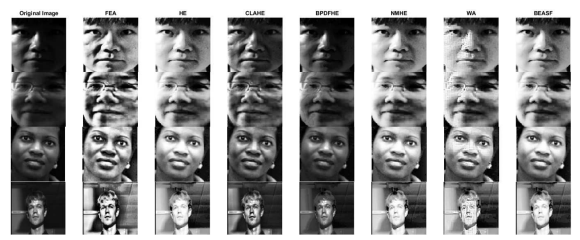

Fig. 8: Example of slight dark face image enhancement using our approach (FEA), HE, CLAHE, BPDFHE, NMHE, WA and BEASF. From the top row to the bottom row, face samples belong to EYale-B, Mobio, FERET and CMU-PIE, respectively.

In term of slight brightness Figure 8 or images with uniform illumination, all the approaches fails to provide a significant improvement except the CLAHE and HE, where better face details and features appear. 


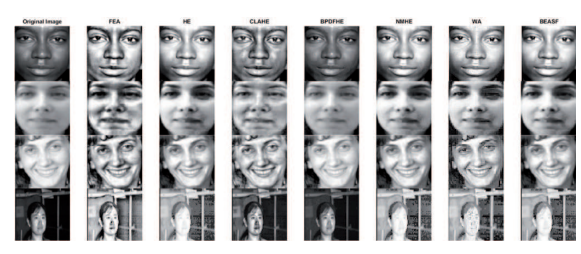

Fig. 9: Example of slight bright face image enhancement using our approach (FEA), HE, CLAHE, BPDFHE, NMHE, WA and BEASF. From the top row to the bottom row, face samples belong to EYale-B, Mobio, FERET and CMU-PIE, respectively.

Another observation where the input faces are too bright is presented in Figure 9. HE, NMHE and BEASF reduce over-lightning, where the BPDFHE almost unchanged the original image.

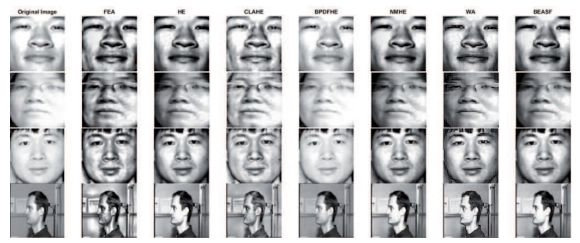

Fig. 10: Example of brightest face image enhancement using our approach (FEA), HE, CLAHE, BPDFHE, NMHE, WA and BEASF. From the top row to the bottom row, face samples belong to EYale-B, Mobio, FERET and CMU-PIE, respectively..

All the illumination variations (darkest to lightest) over the four data sets reflect that the proposed approach tends to give the best visual enhancement by preserving face details and bringing out more details regardless the lightning conditions on the other hand. Indeed, visual inspection confirms the quantitative assessment showing that our approach increases the sharpness and the contrast of the face images.

\section{Conclusions}

This paper proposed an innovative approach for facial image enhancement using Fuzzy theory. An adaptive factor is computed by Fuzzy inference based on the original image illumination, and used for correcting and enhancing the illumination. We performed a rigorous evaluation of the proposed approach on four challenging face data sets, providing a fair comparison to the state-of-the-art. To this end, both qualitative and quantitative assessments are investigated. The evaluation demonstrates that our approach achieves the highest qualitative and quantitative improvements compared to those obtained through other techniques. This is mainly due to the fact that our approach effectively improves the local face contrast and sharpness in both bright 
and dark regions, regardless of the illumination conditions. As a future work, we intend to evaluate the proposed enhancement technique for automatic face recognition under severe illumination conditions.

\section{Acknowledgments}

This research received funding from the Algerian Ministry of Higher Education and Scientific Research (AMHESR) via the National Research Fund project (AVVISAFNR-2013-2016/003).

\section{References}

1. Agaian, S., Roopaei, M., Shadaram, M., Bagalkot, S.S.: Bright and dark distance-based image decomposition and enhancement. In: 2014 IEEE International Conference on Imaging Systems and Techniques (IST) Proceedings, pp. 73-78 (2014). DOI 10.1109/IST.2014.6958449

2. Agaian, S.S., Panetta, K., Grigoryan, A.M.: A new measure of image enhancement. In: IASTED International Conference on Signal Processing \& Communication, pp. 19-22. Citeseer (2000)

3. Agaian, S.S., Panetta, K., Grigoryan, A.M.: Transform-based image enhancement algorithms with performance measure. IEEE Transactions on Image Processing 10(3), 367-382 (2001). DOI 10. $1109 / 83.908502$

4. Arriaga-Garcia, E.F., Sanchez-Yanez, R.E., Garcia-Hernandez, M.G.: Image enhancement using bihistogram equalization with adaptive sigmoid functions. In: Electronics, Communications and Computers (CONIELECOMP), 2014 International Conference on, pp. 28-34 (2014). DOI 10.1109/ CONIELECOMP.2014.6808563

5. Chang, S.J., Li, S., Andreasen, A., Sha, X.Z., Zhai, X.Y.: A reference-free method for brightness compensation and contrast enhancement of micrographs of serial sections. PloS one 10(5), e0127,855 (2015)

6. Du, S., Ward, R.: Wavelet-based illumination normalization for face recognition. In: IEEE International Conference on Image Processing 2005, vol. 2, pp. II-954-7 (2005). DOI 10.1109/ICIP.2005. 1530215

7. Eramian, M., Mould, D.: Histogram equalization using neighborhood metrics. In: The 2nd Canadian Conference on Computer and Robot Vision (CRV'05), pp. 397-404. IEEE (2005)

8. Georghiades, A., Belhumeur, P., Kriegman, D.: From few to many: Illumination cone models for face recognition under variable lighting and pose. IEEE Trans. Pattern Anal. Mach. Intelligence 23(6), 643-660 (2001)

9. Kryszczuk, K., Drygajlo, A.: On face image quality measures. In: Proceedings of the 2nd Workshop on Multimodal User Authentication (2006)

10. Lee, K.C., Ho, J., Kriegman, D.J.: Acquiring linear subspaces for face recognition under variable lighting. IEEE Transactions on pattern analysis and machine intelligence 27(5), 684-698 (2005)

11. McCool, C., Marcel, S., Hadid, A., Pietikainen, M., Matejka, P., Cernocky, J., Poh, N., Kittler, J., Larcher, A., Levy, C., Matrouf, D., Bonastre, J.F., Tresadern, P., Cootes, T.: Bi-modal person recognition on a mobile phone: using mobile phone data. In: IEEE ICME Workshop on Hot Topics in Mobile Multimedia (2012)

12. Phillips, P.J., Wechsler, H., Huang, J., and, P.J.R.: The feret database and evaluation procedure for face-recognition algorithms. Image Vision Comput. pp. 295-306 (1998)

13. Pizer, S., Amburn, E., Austin, J., Cromartie, R., Geselowitz, A., Greer, T., ter Haar Romeny, B., Zimmerman, J., Zuiderveld, K.: Adaptive histogram equalization and its variations. Computer Vision, Graphics, and Image Processing 39(3), 355-368 (1987)

14. Poddar, S., Tewary, S., Sharma, D., Karar, V., Ghosh, A., Pal, S.K.: Non-parametric modified histogram equalisation for contrast enhancement. IET Image Processing 7(7), 641-652 (2013). DOI 10.1049/iet-ipr.2012.0507

15. Roopaei, M., Agaian, S., Shadaram, M., Hurtado, F.: Cross-entropy histogram equalization. In: 2014 IEEE International Conference on Systems, Man, and Cybernetics (SMC), pp. 158-163 (2014). DOI 10.1109/SMC.2014.6973900 
16. Sheet, D., Garud, H., Suveer, A., Mahadevappa, M., Chatterjee, J.: Brightness preserving dynamic fuzzy histogram equalization. IEEE Transactions on Consumer Electronics 56(4), 2475-2480 (2010). DOI 10.1109/TCE.2010.5681130

17. Sim, T., Baker, S., Bsat, M.: The cmu pose, illumination, and expression (pie) database. In: Proceedings of the Fifth IEEE International Conference on Automatic Face and Gesture Recognition, FGR '02, pp. 53-. IEEE Computer Society, Washington, DC, USA (2002). URL http: //dl.acm.org/citation. cfm?id=874061.875452

18. Tizhoosh, H.R.: Fuzzy image enhancement: An overview. In: E.E. Kerre, M. Nachtegael (eds.) Fuzzy Techniques in Image Processing, pp. 137-171. Physica-Verlag HD, Heidelberg (2000). DOI 10.1007/ 978-3-7908-1847-5_5. URL http://dx.doi.org/10.1007/978-3-7908-1847-5_5

19. Yager, R.R., Zadeh, L.A.: Fuzzy Sets, Neural Networks and Soft Computing, 1st edn. John Wiley \& Sons, Inc., New York, NY, USA (1994)

20. Zadeh, L.A.: Outline of a new approach to the analysis of complex systems and decision processes. IEEE Transactions on Systems, Man, and Cybernetics SMC-3(1), 28-44 (1973). DOI 10.1109/TSMC.1973.5408575

21. Zhao, W., Chellappa, R., Phillips, P.J., Rosenfeld, A.: Face recognition: A literature survey. ACM Comput. Surv. 35(4), 399-458 (2003). DOI 10.1145/954339.954342. URL http://doi.acm.org/ 10.1145/954339.954342

22. Zhou, Y., Panetta, K., Agaian, S.: Human visual system based mammogram enhancement and analysis. In: Image Processing Theory Tools and Applications (IPTA), 2010 2nd International Conference on, pp. 229-234. IEEE (2010) 